\title{
MicroRNA-185 suppresses growth and invasion of colon cancer cells through inhibition of the hypoxia-inducible factor-2 $\alpha$ pathway in vitro and in vivo
}

\author{
ZHAN-JUN LU ${ }^{1}$, LUN-GEN LU ${ }^{1}$, KAI-ZHONG TAO ${ }^{1}$, DA-FAN CHEN ${ }^{1}$, QING XIA ${ }^{1}$, \\ JIAN-JUN WENG ${ }^{1}$, FENG ZHU ${ }^{1}$, XING-PENG WANG ${ }^{1}$ and PING ZHENG ${ }^{2}$ \\ ${ }^{1}$ Department of Gastroenterology, Shanghai First People's Hospital, \\ Shanghai Jiao Tong University School of Medicine, Shanghai 200080; \\ ${ }^{2}$ Department of Gastroenterology, Shanghai East Hospital Affiliated to Tongji University, \\ Shanghai 200120, P.R. China
}

Received October 9, 2013; Accepted November 14, 2013

DOI: $10.3892 / \mathrm{mmr} .2014 .2562$

\begin{abstract}
MicroRNAs (miRs) are small non-coding RNAs with regulatory roles, which are involved in a broad spectrum of physiological and pathological processes, including cancer development and progression. However, the function of miR-185 in the development of human colon cancer has not yet been investigated. In this study, the association between miR-185 expression and the clinicopathological characteristics of patients with colon cancer was analyzed using quantitative polymerase chain reaction (qPCR). Using a gain-of-function approach, the effects of miR-185 overexpression on the expression of hypoxia-inducible factor-2 $\alpha$ (HIF-2 $\alpha$ ), proliferating cell nuclear antigen (PCNA) and matrix metallopeptidase-2 (MMP-2) were investigated in SW620 colon cancer cells using qPCR and western blotting. Functional analysis of cellular proliferative activities, by MTT assay, and invasive potential, by Transwell assay, was conducted on SW620 cells expressing low levels of miR-185. miR-185 was found to be significantly downregulated in cancer tissues compared with adjacent non-cancerous tissues, and was negatively correlated with lymph node metastasis of colon cancer $(\mathrm{P}<0.001)$. miR-185 overexpression in vitro impeded cellular proliferation and invasive potential with reduced expression of HIF- $2 \alpha$, PCNA and MMP-2 in SW620 cells transfected with an miR-185 mimic. In addition, the tumor volumes in SW620 subcutaneous nude mouse models treated with miR-185 were significantly smaller than those of the control group. In conclusion, these findings indicate that miR-185 as a tumor suppressor may affect the
\end{abstract}

Correspondence to: Professor Ping Zheng, Department of Gastroenterology, Shanghai East Hospital Affiliated to Tongji University, 150 Jimo Road, Shanghai 200120, P.R. China

E-mail: zhangping201305@163.com

Key words: microRNA-185, hypoxia-inducible factor- $2 \alpha$, colon cancer, proliferation, invasion development of colon cancer cells via inhibition of HIF- $2 \alpha$ signaling, suggesting that miR-185 may serve as a potential therapeutic target in cancer treatment.

\section{Introduction}

Colorectal cancer (CRC) is a major burden to healthcare systems worldwide, accounting for approximately one million novel cancer cases (1). Although CRC mortality has decreased over the last 20 years, it remains the third most common cause of cancer-associated mortality, accounting for $\sim 600,000$ fatalities in 2008 worldwide (1). Therefore, identification of tumor biomarkers for screening and early detection is imperative for patients with colon cancer.

MicroRNAs (miRs) are endogenous small non-coding RNA molecules that regulate gene expression in a sequence-specific manner. This is primarily accomplished through binding to the 3' untranslated region of target mRNAs, either targeting the transcripts for degradation or inhibiting their translation (2). miRs are crucial not only in tissue development but also in the pathology of a number of diseases. A reduction in miR-185 expression levels has been demonstrated to alter dendritic and spinal development, and has also been implicated in psychiatric disorders and cognitive dysfunction (3). miR-185 regulates numerous biological functions, including immune and inflammatory responses, and glutathione metabolism in alcoholic liver disease (4). miR-185 also represses selective high-density lipoprotein cholesterol uptake through the inhibition of scavenger receptor class B member 1 in human hepatic cells, implicating an important role of miRs in modulating cholesterol metabolism (5).

The deregulation of miR expression has been identified in several types of cancer. miR-185 was observed to exhibit significant differential expression in prostate cancer and normal tissue, and has been shown to suppress the proliferation, invasion, migration and tumorigenicity of prostate cancer cells through targeting androgen receptors (6). Furthermore, miR-185 was found to inhibit cell growth and cell cycle progression, and its expression was reduced in non-small cell 
lung cancer (7). However, miR microarray analysis revealed upregulation of miR-185 expression in gastric and bladder cancer, which was validated by quantitative polymerase chain reaction (qPCR), determining a novel stimulatory function of miR-185 in cancer $(8,9)$. In order to investigate the function of miR-185 in colon cancer, the clinical significance of miR-185 expression in colon cancer was analyzed in the present study using qPCR, and the effects of miR-185 overexpression on cell proliferation and the invasive potential of SW620 colon cancer cells were investigated, in order to evaluate miR-185 as a potential therapeutic target in colon cancer.

\section{Materials and methods}

Materials. The SW620, SW480, LOVO, HCT8 and HT29 human colon cancer cell lines used in the experiments was obtained from the Institute of Biochemistry and Cell Biology (Shanghai, China). The colon cancer tissue and the corresponding adjacent non-cancerous tissues (ANCT) were collected from 30 patients at the Department of General Surgery of Shanghai First People's Hospital, Affiliated to Shanghai Jiaotong University (Shanghai, China). This study was approved by the Medical Ethics Committee of Shanghai Jiao Tong University (Shanghai, China) and written informed consent was obtained from the patients or their parents prior to sample collection. Two pathologists reviewed each of the cases. miR-185 mimic and negative control vectors were provided by Shanghai Genechem Co., Ltd. (Shanghai, China). The miR-185 and HIF-2 $\alpha$ primers were synthesized by Applied Biosystems (Foster City, CA, USA). All antibodies were provided by Santa Cruz Biotechnology, Inc. (Santa Cruz, CA, USA).

Drugs and reagents. Dulbecco's modified Eagle's medium (DMEM) and fetal bovine serum (FBS) were purchased from Thermo Fisher Scientific Inc (Waltham, MA, USA); TRIzol Reagent and Lipofectamine 2000 were from Invitrogen Life Technologies (Carlsbad, CA, USA); M-MLV Reverse Transcriptase was from Promega GmbH (Madison, WI, USA); SYBR Green Master Mixture was from Takara Bio, Inc. (Shiga, Japan). The Enhanced Chemiluminescence (ECL)-PLUS kit was from GE Healthcare (Piscataway, NJ, USA).

Reverse-transcription (RT) and $q P C R$. RT-PCR was used to detect the expression of the primary transcripts and mature products of miR-185, and qPCR was used to determine the expression of miR-185 and HIF-2 $\alpha$. Briefly, for the primary transcript, $11 \mu \mathrm{g}$ total RNA was reversely transcribed using oligo-dT primer (Takara Bio, Inc.), and $2 \mu 1$ reverse transcription reaction mix was amplified by PCR as follows: Denaturation at $95^{\circ} \mathrm{C}$ for $2 \mathrm{~min}$ and 25 cycles at $95^{\circ} \mathrm{C}$ for $30 \mathrm{sec}, 55^{\circ} \mathrm{C}$ for $30 \mathrm{sec}$ and $72^{\circ} \mathrm{C}$ for $1 \mathrm{~min}$. For the mature product, $1 \mu \mathrm{g}$ total RNA was reverse-transcribed using miR-185-specific stem-loop RT primer, and $2 \mu$ reverse transcription mix was amplified by PCR as follows: Denaturation at $95^{\circ} \mathrm{C}$ for 2 min and 25 cycles (semiquantitative RT-PCR) or 50 cycles (quantitative real-time PCR) at $95^{\circ} \mathrm{C}$ for $10 \mathrm{sec}$ and $60^{\circ} \mathrm{C}$ for $1 \mathrm{~min}$. The average level of U6 small nuclear (sn) RNA served as an internal control. The SYBR green (Takara Bio, Inc.) method and the IQ5 Real-time PCR detection system (Bio-Rad, Hercules, CA) were used for qPCR. The primer sequences for the qPCR were as follows: Forward: 5'-CAATGGAGAGAAAGGCAGTTCC-3' and reverse: 5'-AATCCATGAGAGATCCCTACCG-3' for miR-185; forward: 5'-ATTGGAACGATACAGAGAAGATT-3' and reverse: 5'-GGAACGCTTCACGAATTTG-3' for U6 snRNA; forward: 5'-GCGCTAGACTCCGAGAACAT-3' and reverse: 5'-TGGCCACTTACTACCTGACCCTT-3' for HIF-2 $\alpha$; and forward: 5'-CAACGAATTTGGCTACAGCA-3' and reverse 5'-AGGGGTCTACATGGCAACTG-3' for GAPDH.

Cell culture and infection. The SW620 cells were cultured in DMEM supplemented with $10 \%$ heat-inactivated FBS, $100 \mathrm{U} / \mathrm{ml}$ penicillin and $100 \mu \mathrm{g} / \mathrm{ml}$ streptomycin. This was placed in a humidified atmosphere containing $5 \% \mathrm{CO}_{2}$ at $37^{\circ} \mathrm{C}$. Either the miR-185 mimic or the negative control was used to infect the SW620 cells. The cells were subcultured at a 1:5 dilution in $300 \mu \mathrm{g} / \mathrm{ml} \mathrm{G} 418$-containing medium. Positive stable transfectants were selected and expanded for further investigation. The clone transfected by the miR-185 mimic was termed the miR-185 mimic group, the clone transfected by the negative control was termed the NC group and SW620 cells without transfection were termed the CON group.

Western blot analysis. Colon cancer cells were harvested and extracted using lysis buffer (containing Tris-HCl, SDS, mercaptoethanol and glycerol). The cell extracts were boiled for $5 \mathrm{~min}$ in loading buffer and then equal quantities of cell extracts were separated on $15 \%$ SDS-PAGE gels. The separated protein bands were transferred to polyvinylidene fluoride membranes and the membranes were then blocked in 5\% skimmed milk powder. The primary antibodies against HIF-2 $\alpha$, proliferating cell nuclear antigen (PCNA) and matrix metallopeptidase-2 (MMP-2) were diluted according to the manufacturer's instructions and incubated overnight at $4^{\circ} \mathrm{C}$. Horseradish peroxidase-linked goat anti-mouse and goat anti-rabbit IgG secondary antibodies were then added at a dilution ratio of 1:1,000 and the membranes were incubated at room temperature for $2 \mathrm{~h}$. The membranes were then washed with phosphate-buffered saline (PBS) three times and the immunoreactive bands were visualized using the ECL-PLUS kit according to the manufacturer's instructions. The relative protein levels of the different cell lines were normalized to the GAPDH concentration. Three separate experiments were performed for each clone.

Cell proliferation assay. Cell proliferation was analyzed with the MTT assay. Briefly, cells infected with miR-185 mimic were incubated in 96-well-plates at a density of $1 \times 10^{5}$ cells per well in DMEM supplemented with $10 \%$ FBS. The cells were treated with $20 \mu \mathrm{l}$ MTT dye for $0,24,48$ and $72 \mathrm{~h}$, and then incubated with $150 \mu \mathrm{l}$ dimethylsulfoxide for $5 \mathrm{~min}$. The color reaction was measured at $570 \mathrm{~nm}$ with an Automated immunoassay analyzer (Bio-Rad Laboratories, Hercules, CA, USA). The proliferation activity was calculated for each clone.

Transwell invasion assay. Transwell filters were coated with Matrigel (BD Biosciences, Franklin Lakes, NJ, USA; $3.9 \mu \mathrm{g} / \mu \mathrm{l}$, 60-80 $\mu \mathrm{l}$ ) on the upper surface of a polycarbonic membrane (diameter, $6.5 \mathrm{~mm}$; pore size, $8 \mu \mathrm{m}$; Corning, Corning, NY, 
USA). Following incubation at $37^{\circ} \mathrm{C}$ for $30 \mathrm{~min}$, the Matrigel solidified and served as the extracellular matrix for analysis of tumor cell invasion. Harvested cells $\left(1 \times 10^{5}\right)$ in $100 \mu 1$ serum-free DMEM were added into the upper compartment of the chamber. A total of $200 \mu \mathrm{l}$ conditioned medium derived from NIH3T3 cells served as a source of chemoattractant and was placed in the bottom compartment of the chamber. After $24 \mathrm{~h}$ incubation at $37^{\circ} \mathrm{C}$ with $5 \% \mathrm{CO}_{2}$, the medium was removed from the upper chamber. The non-invaded cells on the upper side of the chamber were scraped off with a cotton swab. The cells that had migrated from the Matrigel into the pores of the inserted filter were fixed with $100 \%$ methanol, stained with hematoxylin, mounted and dried at $80^{\circ} \mathrm{C}$ for $30 \mathrm{~min}$. The number of cells invading through the Matrigel was counted in three randomly selected visual fields from the central and peripheral portions of the filter using an inverted microscope (Olympus, Tokyo, Japan; magnification, x200). Each assay was repeated three times.

Subcutaneous tumor model and gene therapy. Female immune-deficient nude mice (BALB/c-nu; age, 6 weeks) from the laboratory animal facility of the Hematology Institute of Chinese Academy of Sciences (Shanghai, China) were housed individually in microisolator ventilated cages with free access to water and food. All experimental procedures were performed according to the regulations and internal biosafety and bioethics guidelines of Shanghai Tongji University and the Shanghai Municipal Science and Technology Commission (Shanghai, China).

Four mice were injected subcutaneously with $1 \times 10^{8}$ SW620 cells in $50 \mu$ PBS pre-mixed with an equal volume of matrigel matrix (Becton-Dickinson, Franklin Lakes, NJ, USA). The mice were monitored daily and three out of the four mice developed a subcutaneous tumor. When the tumors reached $\sim 5 \mathrm{~mm}$ in length, they were surgically removed, cut into $1-2-\mathrm{mm}^{3}$ sections and reseeded individually into six other mice. When the reseeded tumors reached $\sim 5 \mathrm{~mm}$ in length, the mice were randomly assigned to either the NC group or the miR-185-treated group. In the miR-185 group, $15 \mu \mathrm{l}$ lentivirus was injected into the subcutaneous tumors using a multi-site injection format. The mice in the NC group received $15 \mu \mathrm{l}$ PBS only. Injections were repeated every other day following the initial treatment. The tumor volume was measured every three days with a caliper, using the formula: Volume $=(\text { length } \mathrm{x} \text { width })^{2} / 2$.

Statistical analysis. SPSS 21.0 software (IBM, Armonk, NY, USA) was used for the statistical analysis. One-way analysis of variance (ANOVA) was used to analyze the differences between groups. The least significant difference method of multiple comparisons was used when the probability for ANOVA was statistically significant and $\mathrm{P}<0.05$ was considered to indicate a statistically significant difference.

\section{Results}

Correlation of miR-185 expression with clinicopathologic characteristics of patients with colon cancer. The expression of miR-185 in colon cancer tissues and ANCT was evaluated by RT-PCR. As shown in Fig. 1, miR-185 expression was
A

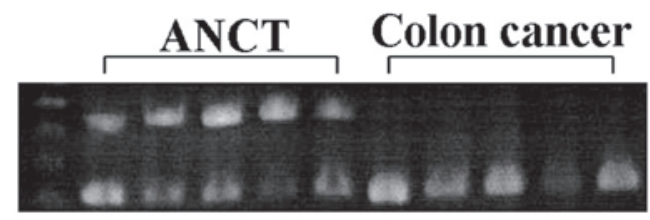

B

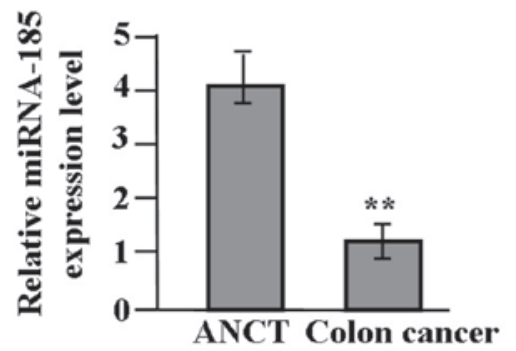

Figure 1. Expression level of miR-185 in colon cancer. (A,B) The expression level of miR-185 was evaluated by quantitative polymerase chain reaction in human colon cancer tissues and adjacent non-cancerous tissues (ANCT), and was found to be significantly reduced in cancer tissues compared with the $\operatorname{ANCT}(* * \mathrm{P}<0.01)$.

significantly reduced in cancer tissues compared with the ANCT $(\mathrm{P}<0.01)$.

The correlation of miR-185 expression with clinicopathologic characteristics of patients with colon cancer was further analyzed. As shown in Table I, the reduced expression of miR-185 was closely associated with lymph node metastasis of colon cancer $(\mathrm{P}<0.001)$, but was not associated with the age or gender of the patients, tumor size, degree of tumor differentiation or tumor-node-metastasis stage $(\mathrm{P}>0.05)$.

Modulation of miR-185 and HIF-2 $\alpha$ expression. The expression of miR-185 and HIF-2 $\alpha$ was examined in different colon cancer cell lines. The expression level of miR-185, indicated by real-time PCR, was significantly reduced (Fig. 2A), while that of HIF-2 $\alpha$ was increased in the SW620 colon cancer cells compared with the other cell lines, as shown by western blot analysis (Fig. 2B and C).

To monitor the effect of miR-185 overexpression on HIF- $2 \alpha$ expression in target cells expressing low levels of miR-185, the miR-185 mimic and scrambled control were delivered into SW620 cells. The expression levels of miR-185 and HIF-2 $\alpha$ following miR-185 mimic infection were further examined for $48 \mathrm{~h}$ by qPCR. As shown in Fig. 3A and B, a significant increase in miR-185 expression and a significant reduction in HIF- $2 \alpha$ mRNA expression respectively were observed in the miR-185 mimic group compared with the $\mathrm{NC}$ and $\mathrm{CON}$ groups (each $\mathrm{P}<0.01$ ). The expression level of HIF- $2 \alpha$ protein, indicated by western blot analysis, was significantly downregulated in the miR-185 mimic group in comparison with the NC and CON groups $(\mathrm{P}<0.01$, Fig. $3 \mathrm{C}$ and $\mathrm{D})$.

Effect of miR-185 overexpression on cell proliferation. Deregulated cell proliferation is a hallmark of cancer (10). In order to analyze the effect of miR-185 overexpression on the growth of colon cancer cells, the proliferative activities of SW620 cells were investigated by an MTT assay. miR-185 overexpression significantly diminished the proliferative activities of SW620 cells compared with the NC and CON groups $(\mathrm{P}<0.01$, 
Table I. Correlation of miR-185 expression with clinicopathologic characteristics of patients with colon cancer.

Variable

Cases (n)

Relative expression

level of miR-185

Age (years)

$(95 \% \mathrm{CI})$

P-value

$\geq 60$

$<60$

Sex

Male

Female

Tumor size $(\mathrm{cm})$

$\geq 5$

$<5$

Degree of differentiation

Well/Moderately

Poorly

TNM stage

I+II

III+IV

Lymph node metastasis

Negative

Positive

\section{6}

14

$0.36-22.35$

$0.32-32.67$

18

12

$0.35-35.78$

$0.32-24.37$

20

10

$0.32-35.47$

$0.37-22.15$

22

0.36-22.35

0.36-22.35

0.36-36.47

0.32-32.77

0.39-37.67

$0.31-9.12$

CI, confidence interval; miR, microRNA; TNM, tumor node metastasis.

A

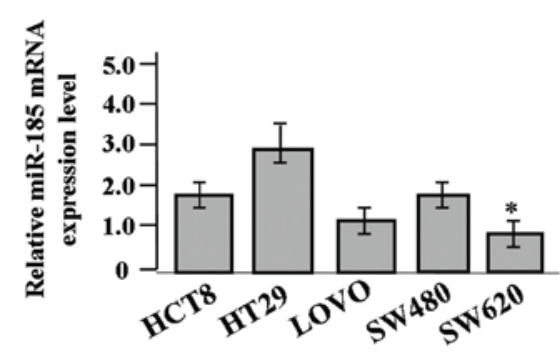

B

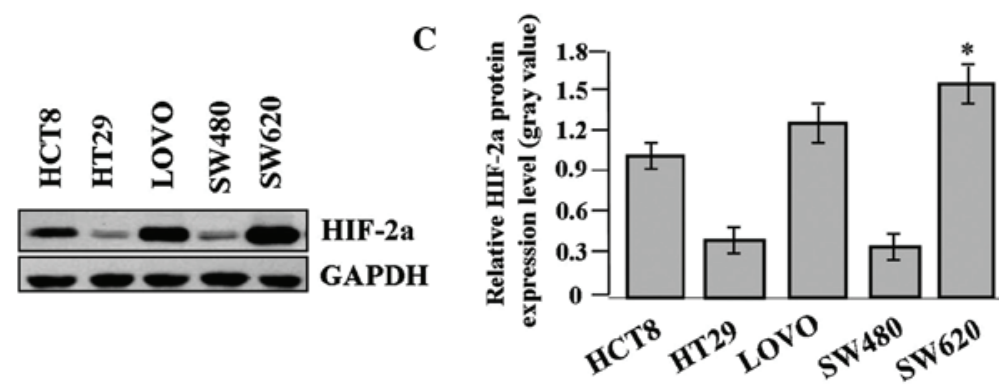

Figure 2. Expression of microRNA (miR)-185 and hypoxia-inducible factor (HIF)-2 $\alpha$ in different colon cancer cell lines. The expression levels of miR-185 and HIF-2 $\alpha$ were respectively examined in different colon cancer cell lines by quantitative polymerase chain reaction and western blotting. (A) The expression level of miR-185 was significantly reduced ( $\mathrm{P}<0.01)$, but (B and C) the expression level of HIF-2 $\alpha$ was increased (* $\mathrm{P}<0.01)$ in $\mathrm{SW620}$ colon cancer cells compared with the other cell lines.

Fig. 4A). In order to determine whether miR-185 suppressed the endogenous expression of PCNA through translational repression, the expression of PCNA protein was examined by western blot analysis, which indicated that the quantity of
PCNA protein was significantly reduced in the miR-185 mimic group compared with the $\mathrm{NC}$ and $\mathrm{CON}$ groups $(\mathrm{P}<0.01$, Fig. 4B and C), suggesting that miR-185 may inhibit the proliferation of colon cancer cells through downregulation of PCNA expression. 
A

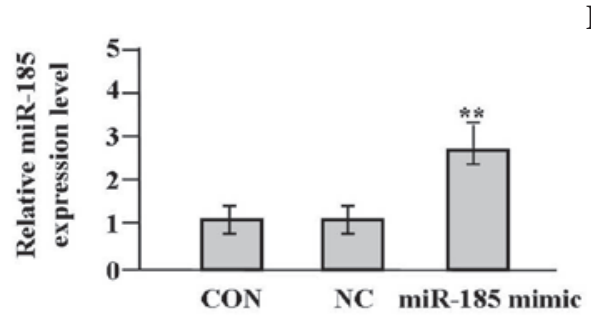

$\mathbf{C}$

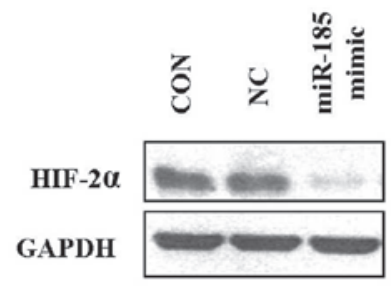

B

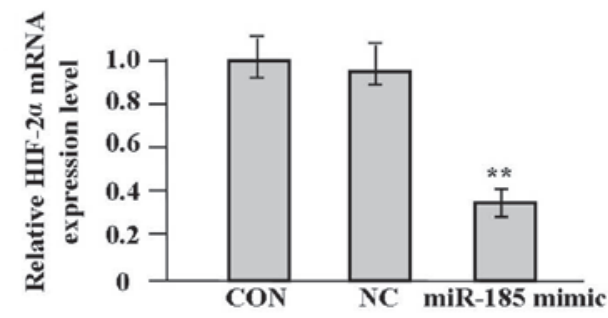

D

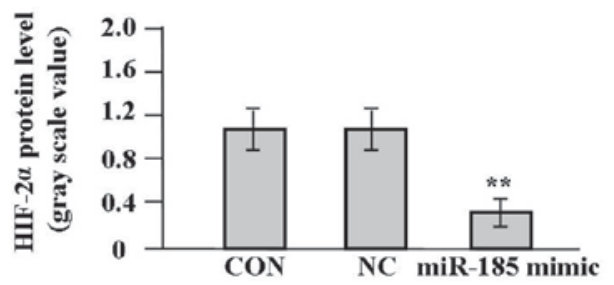

Figure 3. MicroRNA (miR)-185 and hypoxia-inducible factor (HIF)-2 $\alpha$ expression in SW620 cells. (A and B) Quantitative polymerase chain reaction assay indicated a significant increase in miR-185 expression, but a significant reduction in HIF-2 $\alpha$ expression levels in the miR-185 mimic group compared with the negative control $(\mathrm{NC})$ and no transfection $(\mathrm{CON})$ groups $\left({ }^{* *} \mathrm{P}<0.01\right)$. (C and $\left.\mathrm{D}\right)$ Western blot analysis indicated reduced expression levels of HIF-2 $\alpha$ protein in the miR-185 mimic group in comparison with the $\mathrm{NC}$ and $\mathrm{CON}$ groups $\left({ }^{* *} \mathrm{P}<0.01\right)$.

A

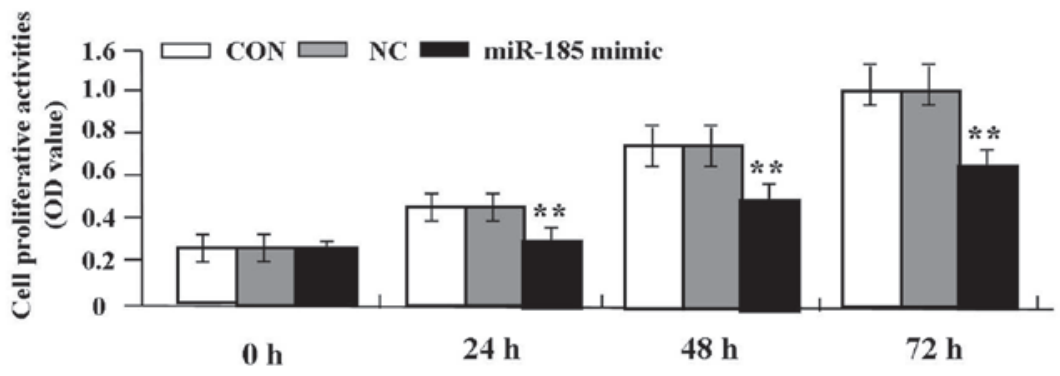

B

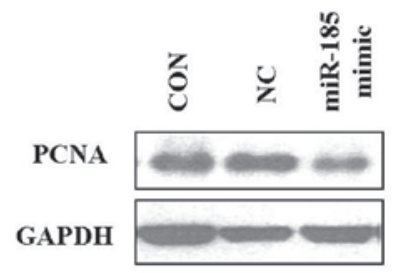

C

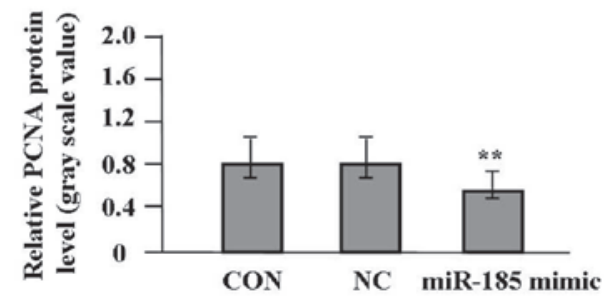

Figure 4. Effect of microRNA (miR)-185 overexpression on cell proliferation. (A) miR-185 overexpression significantly inhibited the proliferative activities of SW620 colon cancer cells in a time-dependent manner compared with the negative control (NC) and no transfection (CON) groups ("* P<0.01). (B and C) Western blot analysis indicated reduced expression levels of proliferating cell nuclear antigen (PCNA) protein in the miR-185 mimic group in comparison with the NC and CON groups in SW620 cells $\left({ }^{* *} \mathrm{P}<0.01\right)$.

Effect of miR-185 overexpression on cell invasion. To determine the effect of miR-185 overexpression on the invasive potential of colon cancer cells, a Transwell assay was performed. The invasive and metastatic potential was determined in the Transwell assay on the basis of the ability of cells to invade a matrix barrier containing laminin and type IV collagen, the predominant components of the basement membrane. Representative micrographs of Transwell filters are shown in Fig. 5A. The invasive potential of SW620 cells was significantly reduced in the miR-185 mimic group compared with the $\mathrm{NC}$ and $\mathrm{CON}$ groups $(\mathrm{P}<0.01$, Fig. $5 \mathrm{~B})$. A western blot analysis was performed to investigate the effect of miR-185 overexpression on the endogenous expression of the MMP-2 protein. The expression level of MMP-2 was found to be significantly reduced in the miR-185 mimic group compared with the $\mathrm{NC}$ and $\mathrm{CON}$ groups $(\mathrm{P}<0.01$, Fig. $5 \mathrm{C}$ and D), indicating that miR-185 may inhibit cell invasion of colon cancer through downregulation of MMP-2 expression.

Antitumor effect of miR-185 in the SW620 xenograft model. The in vitro experiments demonstrated that overexpression of miR-185 efficiently inhibited proliferation and invasion in SW620 colon cancer cells. The antitumor effect of miR-185 was further investigated in vivo using the SW620 xenograft model and lentivirus-mediated gene therapy. The mean volume of tumors in all experimental mice prior to treatment was 
A

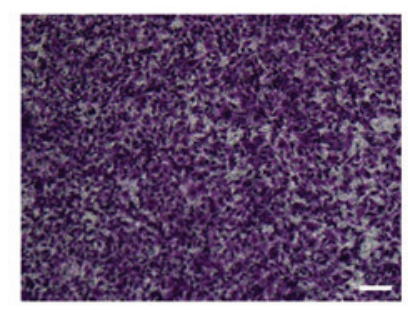

B

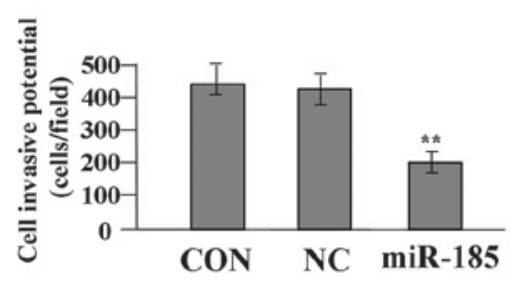

NC

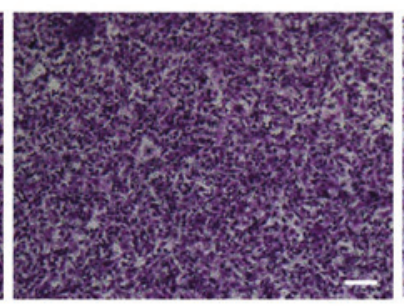

C

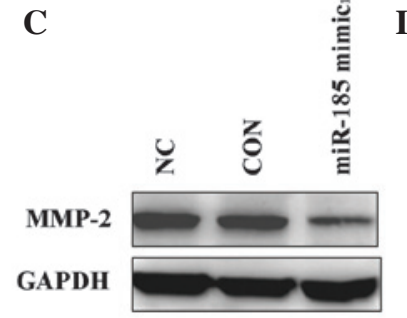

miR-185 mimic

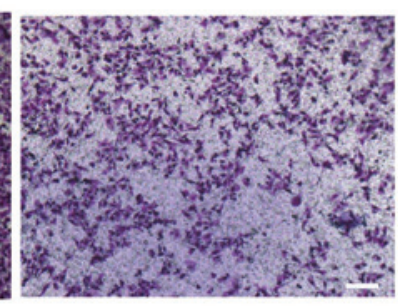

D

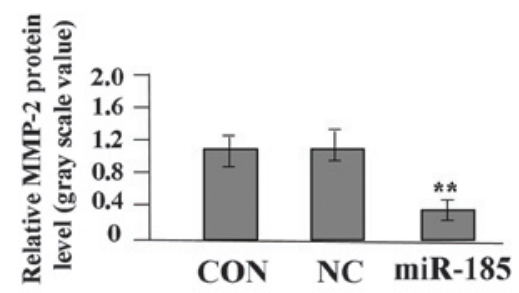

Figure 5. Effect of microRNA (miR)-185 overexpression on cell invasion. (A and B) Cell invasion was determined by Transwell assay, indicating that the invasive potential of colon cancer cells was distinctly reduced in the miR-185 mimic group compared with the negative control (NC) and no tranfection (CON) groups ( $\left({ }^{* *} \mathrm{P}<0.01\right)$. (A) Scale bars, $75 \mu \mathrm{m}$. (C and D) Western blot analysis indicated reduced expression of matrix metallopeptidase-2 (MMP-2) protein in the miR-185 mimic group in comparison with the NC and CON groups in SW620 cells (each ${ }^{* *} \mathrm{P}<0.01$ ).

A

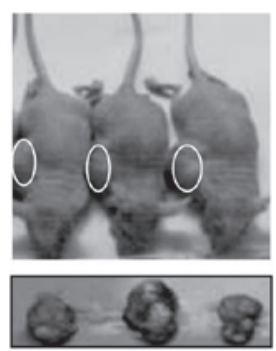

PBS

C

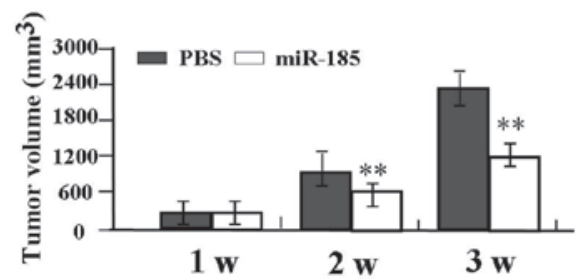

B

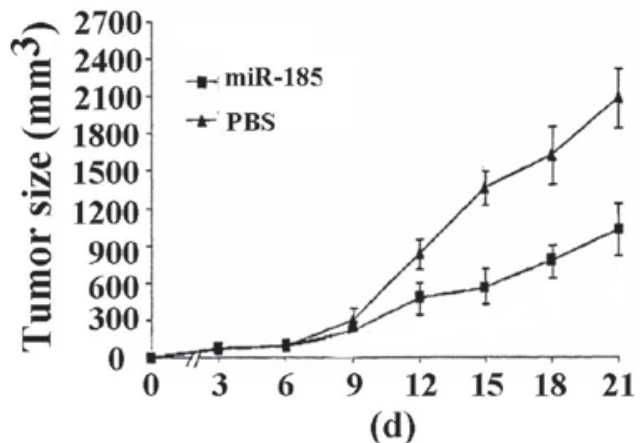

D

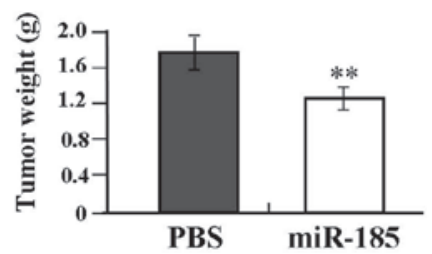

Figure 6. Antitumor effect of microRNA (miR)-185 in the SW620 xenograft model. (A, B) During the first three weeks of recovery, the tumors in the miR-185 group grew at a slower rate compared with the phosphate-buffered saline (PBS) group. (C, D) There was a significant difference in tumor volumes and weight between the miR-185 and PBS groups over the observation period (** $\mathrm{P}<0.01)$. d, days; w, weeks.

$51.65 \pm 8.33 \mathrm{~mm}^{3}$. Each mouse was administered either in situ injection of PBS $(n=3)$ or lentivirus-mediated miR-185 ( $n=3)$. During the first three weeks of recovery, the tumors in the miR-185 group grew at a slower rate compared with those in the PBS group (Fig. 6A and B). There was a significant difference in tumor volumes and weight between the miR-185 and PBS groups over the observation period ( $\mathrm{P}<0.01$, Fig. $6 \mathrm{C}$ and $\mathrm{D})$.

\section{Discussion}

miRs are small regulatory RNAs involved in various physiological and cellular processes. Alterations of miRs are crucial for tumorigenesis (11). miRs exhibit potential as diagnostic and prognostic biomarkers for colon cancer (12). Using miR expression patterns and target prediction, previous studies have observed that miR-185 expression is upregulated and results in loss of function of tumor suppressors in clear cell renal cell carcinoma (13) and lung squamous cell carcinoma (14). However, in the present study, miR-185 was found to have low expression in colon cancer tissues compared with ANCT, suggesting that miR-185 expression may be different among types of tumor tissue. miR-185 expression was demonstrated to be negatively correlated with lymph node metastasis of colon cancer. However, the clinical data collected lacked information regarding the survival times of the colon cancer patients, thus the association between miR-185 expression 
and survival time was not assessed. Nevertheless, another study indicated the potential prognostic value of miR-185 expression levels for predicting clinical outcomes following surgery (15).

Low expression of miR-185 is also associated with a poor outcome in glioma patients. miR-185 was found to suppress glioma cell invasion, indicating that it may be a potential prognostic marker and therapeutic target (16). Previous studies have revealed that miR-185 impedes anchorage-independent growth, cell migration and invasion, in addition to suppressing tumor growth in vivo and increasing cisplatin sensitivity by promoting apoptosis, implicating it as a potent tumor suppressor (17-19). In the present study, overexpression of miR-185 repressed the growth and invasion of colon cancer cells in vitro and in vivo, which was consistent with the results of previous studies, indicating that miR-185 is a negative regulator of RhoA and $\mathrm{Cdc} 42$ and their cellular activities, and can inhibit the proliferation and invasion of CRC cells (20).

A number of studies have shown a link between the microRNA pathway and hypoxia signaling. HIF-2 $\alpha$ is regulated by the Dicer-dependent miR-185, which is downregulated in hypoxia (21). HIF-2 $\alpha$ promotes hypoxic tumor cell proliferation by enhancing c-myc transcriptional activity (22) and results in CRC progression by dysregulating iron homeostasis (23). Disruption of the HIF-2 $\alpha$ gene inhibits tumor angiogenesis and perfusion in human colon cancer cells (24). In the present study, overexpression of miR-185 suppressed proliferation and invasion, and reduced expression levels of HIF- $2 \alpha$ in colon cancer cells, suggesting that miR-185 may be implicated in the development of colon cancer cells through inhibition of the HIF- $2 \alpha$ pathway. However, whether HIF- $2 \alpha$ is the target of miR-185 in colon cancer cells requires further investigation.

The findings of the present study showed that overexpression of miR-185 markedly reduced the expression of PCNA and MMP-2 in colon cancer cells at the translational level, indicating that miR-185 may suppress proliferation and invasion of colon cancer cells via the downregulation of PCNA and MMP-2 expression. PCNA is a nuclear protein that is expressed in proliferating cells and may be required for maintaining cell proliferation, which is used as a marker to evaluate colon cancer cell proliferation $(25,26)$. MMP-2, a predictor of GM cell invasion, is pivotal in the degradation of the extracellular matrix, and thereby enhances the invasive, proliferative and metastatic potential of colon cancer (27). Certain studies have confirmed that HIF-1 signaling promotes tumor migration and invasion through upregulation of MMP-2 and PCNA expression $(28,29)$. However, there was no direct evidence that HIF- $2 \alpha$ promoted proliferation and invasion of colon cancer cells through upregulation of MMP-2 and PCNA expression from the present study. Therefore, miR-185 may be involved in proliferation and invasion of colon cancer cells through inhibition of HIF- $2 \alpha$-mediated PCNA and MMP-2 expression.

In conclusion, these findings indicate that miR-185 as a tumor suppressor may be involved in the development of colon cancer cells via inhibition of HIF-2 $\alpha$ signaling, suggesting that miR-185 may serve as a potential therapeutic target in the treatment of cancer.

\section{Acknowledgements}

This study was supported by the project of Shanghai First People's Hospital (grant no. 12B01).

\section{References}

1. Antonic V, Stojadinovic A, Kester KE, et al. Significance of infectious agents in colorectal cancer development. J Cancer 4: 227-240, 2013.

2. He L and Hannon GJ: MicroRNAs: small RNAs with a big role in gene regulation. Nat Rev Genet 5: 522-531, 2004.

3. Xu B, Hsu PK, Stark KL, et al: Derepression of a neuronal inhibitor due to miRNA dysregulation in a schizophrenia-related microdeletion. Cell 152: 262-275, 2013.

4. Liu Y, Chen SH, Jin X and Li YM: Analysis of differentially expressed genes and microRNAs in alcoholic liver disease. Int $\mathbf{J}$ Mol Med 31: 547-554, 2013.

5. Wang L, Jia XJ, Jiang HJ, et al: MicroRNAs 185, 96, and 223 repress selective high density lipoprotein cholesterol uptake through posttranscriptional inhibition. Mol Cell Biol 33: 1956-1964, 2013

6. Qu F, Cui X, Hong Y, et al: MicroRNA-185 suppresses proliferation, invasion, migration, and tumorigenicity of human prostate cancer cells through targeting androgen receptor. Mol Cell Biochem 377: 121-130, 2013.

7. Takahashi Y, Forrest AR, Maeno E, et al: MiR-107 and MiR-185 can induce cell cycle arrest in human non small cell lung cancer cell lines. PLoS One 4: e6677, 2009.

8. Yao Y, Suo AL, Li ZF, et al: MicroRNA profiling of human gastric cancer. Mol Med Rep 2: 963-970, 2009.

9. Gottardo F, Liu CG, Ferracin M, et al: Micro-RNA profiling in kidney and bladder cancers. Urol Oncol 25: 387-392, 2007.

10. Hanahan D and Weinberg RA: Hallmarks of cancer: the next generation. Cell 144: 646-674, 2011.

11. Mannoor K, Liao J and Jiang F: Small nucleolar RNAs in cancer. Biochim Biophys Acta 1826: 121-128, 2012.

12. Yu G, Tang JQ, Tian ML, et al: Prognostic values of the miR-17-92 cluster and its paralogs in colon cancer. J Surg Oncol 106: 232-237, 2012.

13. Liu H, Brannon AR, Reddy AR, et al: Identifying mRNA targets of microRNA dysregulated in cancer: with application to clear cell Renal Cell Carcinoma. BMC Syst Biol 4: 51, 2010.

14. Yang Y, Li X, Yang Q, et al: The role of microRNA in human lung squamous cell carcinoma. Cancer Genet Cytogenet 200: 127-133, 2010.

15. Akçakaya P,Ekelund S, Kolosenko I, et al: miR-185 and miR-133b deregulation is associated with overall survival and metastasis in colorectal cancer. Int J Oncol 39: 311-318, 2011.

16. Tang H, Wang Z, Liu X, et al: LRRC4 inhibits glioma cell growth and invasion through a miR-185-dependent pathway. Curr Cancer Drug Targets 12: 1032-1042, 2012.

17. Imam JS, Buddavarapu K, Lee-Chang JS, et al: MicroRNA-185 suppresses tumor growth and progression by targeting the Six1 oncogene in human cancers. Oncogene 29: 4971-4979, 2010.

18. Greenberg E, Hershkovitz L, Itzhaki O, et al: Regulation of cancer aggressive features in melanoma cells by microRNAs. PLoS One 6: e18936, 2011.

19. Xiang Y,Ma N, Wang D, et al: MiR-152 and miR-185 co-contribute to ovarian cancer cells cisplatin sensitivity by targeting DNMT1 directly: a novel epigenetic therapy independent of decitabine. Oncogene 33: 378-386, 2014.

20. Liu M, Lang N, Chen X, et al: miR-185 targets RhoA and Cdc42 expression and inhibits the proliferation potential of human colorectal cells. Cancer Lett 301: 151-160, 2011.

21. Ho JJ, Metcalf JL, Yan MS, et al: Functional importance of Dicer protein in the adaptive cellular response to hypoxia. J Biol Chem 287: 29003-29020, 2012.

22. Gordan JD, Bertout JA, Hu CJ, et al: HIF-2 $\alpha$ promotes hypoxic cell proliferation by enhancing c-myc transcriptional activity. Cancer Cell 11: 335-347, 2007.

23. Xue X, Taylor M, Anderson E, et al: Hypoxia-inducible factor-2 $\alpha$ activation promotes colorectal cancer progression by dysregulating iron homeostasis. Cancer Res 72: 2285-2293, 2012.

24. Burkitt K, Chun SY, Dang DT, et al: Targeting both HIF-1 and HIF-2 in human colon cancer cells improves tumor response to sunitinib treatment. Mol Cancer Ther 8: 1148-1156, 2009. 
25. Naryzhny SN: Proliferating cell nuclear antigen: a proteomics view. Cell Mol Life Sci 65: 3789-3808, 2008.

26. Wilson MS and Schofield PF: Markers to study human colonic cell proliferation. Gut 36: 152, 1995.

27. Baker EA, Bergin FG and Leaper DJ: Matrix metalloproteinases, their tissue inhibitors and colorectal cancer staging. Br J Surg 87: 1215-1221, 2000 .
28. Fujiwara S,NakagawaK,HaradaH,et al: Silencing hypoxia-inducible factor-1 $\alpha$ inhibits cell migration and invasion under hypoxic environment in malignant gliomas. Int J Oncol 30: 793-802, 2007.

29. Nakanishi K, Hiroi S, Tominaga S, et al: Expression of hypoxia-inducible factor-1 $\alpha$ protein predicts survival in patients with transitional cell carcinoma of the upper urinary tract. Clin Cancer Res 11: 2583-2590, 2005. 